\title{
Protein content and lipid profiling of isolated native autophagosomes
}

Daniel Schmitt ${ }^{1}$, Süleyman Bozkurt ${ }^{2}$, Pascale Henning-Domres ${ }^{1}$, Heike Huesmann ${ }^{1}$, Stefan Eimer ${ }^{3}$, Laura Bindila ${ }^{4}$, Georg Tascher², Christian Münch², Christian Behl ${ }^{1}$ and Andreas Kern ${ }^{1^{*}}$

\author{
Affiliations \\ ${ }^{1}$ Institute of Pathobiochemistry, The Autophagy Lab, University Medical Center \\ of the Johannes Gutenberg University, 55128 Mainz, Germany \\ 2 Institute of Biochemistry II, Faculty of Medicine, Goethe University, 60590 \\ Frankfurt am Main, Germany \\ 3 Department of Structural Cell Biology, Institute for Cell Biology and \\ Neuroscience, Goethe University, 60438 Frankfurt am Main, Germany \\ 4 Institute of Physiological Chemistry, Clinical Lipidomics Unit, University \\ Medical Center of the Johannes Gutenberg University, 55128 Mainz, Germany

\section{${ }^{*}$ Corresponding author}

\section{Andreas Kern} \\ Institute of Pathobiochemistry, The Autophagy Lab, University Medical Center \\ of the Johannes Gutenberg University, 55128 Mainz, Germany \\ e-mail: akern@uni-mainz.de
}

Running title: Autophagosomal cargo and lipid profiles

Total characters including spaces: 22.572 


\section{Abstract}

Autophagy is a central eukaryotic catabolic pathway responsible for clearance and recycling of an extensive portfolio of cargoes, which are packed in vesicles, called autophagosomes, and are delivered to lysosomes for degradation. Besides basal autophagy, which constantly degrades cellular material, the pathway is highly responsive to several stress conditions. However, the exact protein content and phospholipid composition of autophagosomes under changing autophagy conditions remain elusive so far. Here, we introduce a FACS-based approach for isolation of native unmanipulated autophagosomes and ensure the quality of the preparations. Employing quantitative proteomics and phospholipidomics, we obtained a profound cargo and lipid profile of autophagosomes purified upon basal autophagy conditions, nutrient deprivation, and proteasome inhibition. Indeed, starvation only mildly affected the content profile, while interference with proteasome activity showed stronger effects and specifically altered autophagosome cargoes. Interestingly, the phospholipid composition of autophagosomes was unaffected by the different treatments. Thus, the novel isolation method enables purification of intact autophagosomes in large quantities and allows protein content and phospholipid profiling without the requirement of exhaustive cellular fractionation or genetic manipulation.

\section{Keywords}

autophagy / autophagosome isolation / autophagosome cargo profiling / autophagosome lipid profiling 


\section{Introduction}

Macroautophagy (hereafter autophagy) is a eukaryotic catabolic pathway responsible for removal and recycling of an extensive portfolio of cytosolic cargoes. Numerous proteins, aggregates, organelles, cellular compartments, or pathogens have been characterized as autophagy substrates, which are packed in vesicles, called autophagosomes, and are delivered to lysosomes for degradation (Mizushima \& Komatsu, 2011).

The engulfment of cargo in vesicles permits its separation and mobilization and is crucial for autophagy-lysosomal degradation. The formation of autophagosomes starts with the double membraned phagophore, which expands around the degradable material until it surrounds it and seals (Lamb et al, 2013). This de novo vesicle synthesis is initiated by activation of the ULK $1 / 2$ kinase complex and the phosphoinositide 3-kinase (PI3-kinase) complex that facilitate the recruitment of proteins and lipids essential for phagophore generation (Mercer et al, 2018; Nascimbeni et al, 2017). Upon nucleation from the ER membrane, the phagophore elongates by addition of lipids. While the exact lipid sources remain to be elucidated, recent studies suggest a direct lipid transfer from the ER via ATG2A/B (Osawa et al, 2019; Valverde et al, 2019). Indeed, in yeast it has been shown that phospholipid $(\mathrm{PL})$ synthesis from free fatty acids is important for efficient autophagosome formation (Schutter et al, 2020).

The elongation of the phagophore is linked to two ubiquitin-like conjugation reactions (Mizushima, 2020). Firstly, ATG12 is conjugated to ATG5, which secondly facilitates the conjugation of Atg8 proteins to the phospholipid phosphatidylethanolamine (PE) (Kabeya et al, 2004; Noda \& Inagaki, 2015). In humans, six Atg8 family members have been identified: MAP1LC3A, MAP1LC3B, MAP1LC3C (shortly LC3A-C), as well as GABARAP, GABARAPL1, and GABARAPL2 (Slobodkin \& Elazar, 2013). Lipidated Atg8 proteins are inserted into both sides of the growing phagophore double membrane and stay attached to mature autophagosomes, facilitating phagophore elongation and closure as well as autophagosome-lysosome fusion (Nguyen et al, 2016; Tsuboyama et al, 2016; Weidberg et al, 2010). 
Additionally, Atg8 proteins provide binding sites for cargo receptors. Autophagy was initially described as a non-selective process that degrades random material of the cytosol to recycle building blocks to respond to changing metabolic requirements. The identification of cargo receptors, though, established a selective part by which specific substrates are directed into the pathway (Khaminets et al, 2016; Stolz et al, 2014). Via its LC3 interacting domain and its ubiquitin binding domain, the cargo receptor p62/SQSTM1 e.g. binds to LC3 and mediates the transfer of selective cargoes into autophagosomes (Gatica et al, 2018; Kirkin et al, 2009; Pankiv et al, 2007).

The formation of autophagosomes is highly dynamic and rapidly adapts to changing cellular conditions. Healthy, unstressed cells are characterized by a basal autophagy rate that constantly degrades cellular material at (comparably) low levels. Various stress situations, such as starvation or imbalances of proteostasis, alter autophagy, resulting in a substantially enhanced cargo degradation (He \& Klionsky, 2009). However, the exact profile of the cellular material that is degraded upon different autophagy conditions is not defined so far. Several studies have investigated autophagy substrates upon autophagosome isolation by elaborate cellular fractionation methods with clear limitations in purity (Dengjel et al, 2012; Gao et al, 2010; Mancias et al, 2014). More recently, autophagosome content was examined by proximity labelling in combination with quantitative proteomics, which requires genetic manipulation of the cell (Le Guerroue et al, 2017; Zellner et al, 2021). Thus, extensive comparative cargo and phospholipid analyses of unmanipulated native human autophagosomes from basal and altered autophagy conditions are missing so far.

Here, we now introduce a FACS (fluorescence activated cell sorting)-based isolation approach to purify large quantities of intact native autophagosomes. We characterized the quality of the isolated autophagic vesicles and conducted quantitative proteomic and phospholipidomic analyses to generate profound autophagosome content profiles and PL compositions upon basal autophagy conditions, nutrient deprivation, and proteasome inhibition. Importantly, starvation only mildly affected the autophagosome cargo content profile, while disturbances of proteasomal degradation showed treatment-specific alterations. 
The PL composition of autophagosomes was unaffected by the different autophagy modulations, emphasizing the requirement for a distinct PL pattern in autophagic vesicles.

\section{Results and Discussion}

\section{Isolation of intact native autophagosomes in large quantities}

We established a protocol for isolation of native unmanipulated autophagosomes, employing antibody-based fluorescence tagging of Atg8 proteins and subsequent sorting via FACS (Figure 1A, Appendix Figure S1A). Lipidated Atg8 proteins are inserted into the outer as well as inner membrane of elongating phagophores and stay attached to mature autophagosomes (Kabeya et al., 2004). Upon mild cell disruption, we incubated the cellular extract with a primary antibody directed against LC3B or GABARAP, followed by treatment with a secondary fluorophore-conjugated antibody. The stable and selective attachment of the fluorophore allowed the specific purification using FACS. The sorting resulted in preparations of approx. 1.000 autophagosomes per $\mu$ PBS and thus allowed their purification in large quantities.

In order to confirm the quality of the isolation, we analyzed the presence of autophagosome proteins within the isolate fraction by methanol/chloroformbased protein precipitation and Western blotting. Upon preparation of autophagic vesicles via antibodies directed against LC3B or all GABARAP isoforms (Figure 1B), we detected the lipidated variants of both Atg8 proteins as well as p62/SQSTM1 within the isolate fractions. But unlipidated Atg8 proteins, which are not bound to autophagosomes, and cytoskeleton proteins, were hardly detectable. They were quantitatively excluded by the centrifugation steps integrated in the protocol and the fluorescence-based sorting (Figure 1A, Appendix Figure S1B).

To examine the specificity of the antibody binding and the quality of the preparations, we quantified the co-localization of antibody-mediated fluorescence signals of two different autophagosome proteins. We employed antibodies directed against LC3B, GABARAP, or all GABARAP isoforms and analyzed the fluorescence signal via FACS (Figure $1 \mathrm{C}+\mathrm{D}$, Appendix Figure S2). Indeed, almost $100 \%$ of GABARAP-positive and all GABARAP-positive 
structures co-localized with the LC3B antibody-mediated fluorescence signal. Focusing on LC3B, the GABARAP signal was found on approx. $33 \%$ and the all GABARAP signal on around $93 \%$ of LC3B-marked autophagosomes, respectively. This indicated that the different GABARAP isoforms not necessarily co-localize on single autophagosomes. However, the vast majority of vesicles was indeed positive for two different autophagosome markers, emphasizing the exceptional quality of the isolation approach. Only a minor fraction of autophagosomes showed exclusively one Atg8 family member, LC3B ( $6 \%$ ) or at least one of the GABARAP isoforms ( $1 \%)$. This can be explained in different ways: (1) insufficient antibody binding; (2) a small fraction of autophagosomes is indeed only decorated by one particular Atg8 family member; (3) besides the association with autophagosomes, LC3B is also linked to non-autophagic vesicles. During LC3-associated phagocytosis LC3B-II is inserted into the single membrane of phagosomes, supporting lysosomal degradation of phagosome content (Sanjuan et al, 2007). However, we treated cells with bafilomycin $A_{1}$ prior to autophagosome preparation, which causes a massive accumulation of autophagosomes within the cell. Thus, LC3associated phagosomes should only represent a minor fraction of vesicles devoid of GABARAP isoforms.

Continuing our quality control, we investigated whether the purified autophagosomes were sealed and used proteinase $\mathrm{K}$ digestion to analyze its impact on p62/SQSTM1 (Figure 2A). In intact vesicles, the cargo receptor is inaccessible and thus protected from degradation (Velikkakath et al, 2012). Indeed, p62/SQSTM1 was not prominently degraded by proteinase $\mathrm{K}$ within the isolate fractions, indicating that the overall majority of autophagosomes was closed. For control, we opened autophagic vesicles mechanically, which resulted in the exhaustive digestion of p62/SQSTM1.

Further analyses of the autophagosome preparations were conducted by microscopy. Differential interference contrast (DIC) microscopy showed vesicular structures of different sizes without evidence of cellular debris or accumulations of other cellular material (Figure 2B). This finding was validated by negative stain electron microscopy, which identified double membraned vesicles (Figure $2 \mathrm{C}$ ). The inner core often showed a granular appearance and 
was filled with darkly stained structures most likely resembling proteinous cargo. Thus, visualization of the isolated vesicles also confirmed the preparation of pure and undamaged autophagosomes.

Autophagic vesicles are described to cover size ranges of 500 to $1.500 \mathrm{~nm}$ in diameter (Mizushima et al, 2002). Size evaluation of the purified autophagosomes by EM demonstrated that they showed expected diameter ranges from $340 \mathrm{~nm}$ to $1.150 \mathrm{~nm}$ (Figure 2D). Interestingly, the autophagic vesicles could be clustered into different size groups. The group with the smallest diameters covered $426 \mathrm{~nm}$ on average, the largest grouped with a mean diameter of $1.034 \mathrm{~nm}$. Covering $42 \%$ of all analyzed vesicles, the most abundant autophagosomes showed a mean size of $651 \mathrm{~nm}$ in diameter.

\section{Protein cargo profiles of native autophagosomes upon basal autophagy conditions, starvation, and proteasome inhibition}

The established FACS-based protocol enabled the isolation of pure unmanipulated autophagosomes at high quantities. The vesicles were not damaged by the preparation, which qualified the isolates for bulk cargo analyses. For protein content profiling, we conducted quantitative LC-MS/MS and analyzed purified autophagosomes upon basal autophagy conditions (full medium), EBSS-mediated starvation, and MG132 treatment.

EBSS treatment causes a rapid increase in autophagic activity to recycle building blocks to compensate the lack of nutrients (He \& Klionsky, 2009). Autophagy is regulated by the MTORC1 complex (Condon \& Sabatini, 2019), which, in its active form, facilitates anabolic pathways and restricts autophagic activity. Upon starvation, MTORC1 is inactivated, resulting in the promotion of catabolic processes and in autophagy induction.

MG132 inhibits the proteasome and triggers an imbalance of proteostasis, causing the accumulation of ubiquitinated proteins within the cell. The proteasome is the degradative active component of the ubiquitin proteasome system (UPS), which, in addition to autophagy, is a prominent cellular degradation pathway (Pohl \& Dikic, 2019). Importantly, autophagy and the UPS are functionally interrelated. Perturbation of the UPS results in the enhanced 
autophagic degradation of proteasome substrates, which is regulated by the cochaperone BAG3 (Gamerdinger et al, 2009).

To confirm the impact of EBSS and MG132 on autophagy, we conducted Western blotting and immunocytochemical analyses (Appendix Figure S3). As expected, EBSS treatment increased autophagic activity, since the flux of LC3B-II was enhanced (Appendix Figure S3A). This was validated by immunocytochemical stainings of LC3B-positive autophagosomes, which accumulated at high levels in comparison to control cells when lysosomal degradation was inhibited by bafilomycin $A_{1}$ (Appendix Figure S3B). For proteasome inhibition, we treated cells for $8 \mathrm{~h}$ with MG132 and added bafilomycin $A_{1}$ in the last two hours. This treatment resulted in a reduced LC3BII flux and a decreased accumulation of LC3B-positive structures in comparison to control cells (Appendix Figure S3). The duration of proteasome inhibition and the resulting disturbance of proteostasis apparently exhausted the autophagic system. In summary, both treatments modulated autophagy and thus potentially altered the cargo profile compared to basal autophagosomes.

The proteomics analysis of basal autophagosomes detected 4.514 cargo proteins in total, including multiple autophagosome proteins (Source data, Appendix Figure S4). Functional clustering of the top 500 cargo proteins demonstrated that basal autophagic protein substrates grouped into prominent cellular pathways, such as protein and RNA metabolism or vesicle trafficking (Figure 3A). Comparative analysis of the three independently performed autophagosome preparations upon basal conditions showed only subtle changes (Appendix Figure S5A), confirming the quality and reliability of each independent autophagosome preparation, protein processing, and quantitative MS.

Investigating autophagosome content upon EBSS treatment detected only minor alterations despite a significant increase in autophagic flux (Figure 3B, Appendix Figure $\mathrm{S} 5 \mathrm{~B}+\mathrm{C}$ ). Interestingly, a subset of proteins with increased autophagosome appearance clustered within the ribosome biogenesis pathway (Appendix Figure S5B), which might reflect the reduction of cellular anabolic activities mediated by inhibition of MTORC1. However, the minor influence of nutrient deprivation on the autophagosome protein content profile emphasizes 
that this condition results in a globally increased turnover of autophagy substrates without selection of particular cargoes. The requirement to rapidly compensate the lack of nutrients apparently restricts the choice of dispensable cellular material.

In contrast to starvation, proteasome inhibition showed a much stronger impact on autophagosome content, resulting in an altered appearance of multiple proteins (Figure 3C). Importantly, network analysis of proteins with an increased autophagosome appearance revealed that a subset grouped within the proteasome pathway (Figure 3D, Appendix Figure S6A), illustrating an enhanced degradation of UPS components upon MG132 treatment. These included multiple proteasome subunits as well as proteins of the ubiquitination process and interestingly also BAG3. Upon proteasome inhibition, BAG3 upregulates autophagy and mediates the enhanced transfer of ubiquitinated cargoes into autophagosomes (Arndt et al, 2010; Gamerdinger et al., 2009). Contrary to the enhanced degradation of UPS components, proteins with reduced autophagosome appearance upon MG132 treatment clustered within the lysosome pathway (Figure 3E, Appendix Figure S6B, Appendix Figure S7), including LAMP proteins and several acidic hydrolases, such as cathepsins or glycosidases. Thus, inhibition of the proteasome altered the autophagosome cargo profile and the observed changes displayed an adequate adaptation to the treatment: components supporting UPS function were degraded at enhanced levels, whereas factors supporting the lysosome showed a reduced turnover.

In conclusion, analysis of autophagosome content resulted in a detailed representation of autophagy cargoes and showed treatment-specific effects. This emphasizes the quality of the autophagosome preparations and highlights the value of the isolation to investigate content profiles from native unmanipulated autophagic vesicles. The data depicts a profound basis for the definition of autophagic substrates under various autophagy conditions.

\section{Phospholipid composition of native autophagosomes}

In addition isolation of autophagic vesicles enabled the detailed investigation of the autophagosome PL composition. Employing targeted multiplex quantitative 
MS, we quantitatively determined different PL species with diverse fatty acid combinations, concerning chain length and saturation level (Figure 4). Importantly, the PL were unaffected by the different autophagy conditions, despite clear alterations in the rate of autophagosome formation (Appendix Figure S3). This indicates the importance of a distinct PL composition to support autophagosome structure and function.

Phosphatidylcholine (PC) is the most abundant PL in mammalian cells and mammalian membranes typically show more than $50 \%$ of this particular lipid, which was also reflected by the detected amount of PC in isolated autophagosomes (Figure 4A). The second most frequent $\mathrm{PL}$ within the autophagosome fraction was sphingomyelin (SM), the only $\mathrm{PL}$ that is not exclusively synthesized within the ER, but is generated from ceramide within the Golgi apparatus. This lipid is commonly found at the Golgi network, the outer leaflet of the plasma membrane, and in the endocytic system (Slotte, 2013). Consistently, besides the ER, the Golgi and endocytic pathways are frequently described to support autophagosome formation (De Tito et al, 2020). The detection of SM in autophagic vesicles connects autophagosome membranes to the Golgi apparatus or Golgi-derived vesicles and is in line with a recent publication showing that $\mathrm{SM}$ is essential for structure and function of double membrane vesicles responsible for viral replication (Gewaid et al, 2020). In addition to their structural role, PLs exhibit also regulatory functions. While present only in lower amounts, PI, PE, and phosphatidylserine (PS) are crucial for efficient autophagosome formation (de la Ballina et al, 2020). Phosphorylated forms of PI generated by the PI3-kinase complex, are linked to phagophore generation and elongation (Nascimbeni et al., 2017). In an ubiquitin-like conjugation reaction, $\mathrm{PE}$ is covalently attached to Atg8 family members and is essential for efficient phagophore maturation and autophagosome function (Noda \& Inagaki, 2015). Recent publications showed that lipids are transferred from the ER to the growing phagophore by the activity of ATG2A/B (Osawa et al., 2019; Valverde et al., 2019) and that the presence of negatively charged PS in liposomes accelerates the ATG2B-mediated lipid transfer (Osawa et al, 2020). 
Concerning fatty acids, the PLs were linked to various combinations. In particular, short chained and (poly-) unsaturated fatty acids were detected at high levels (Figure 4B). Approx. $90 \%$ of PC showed the short fatty acid combination $34: 1$ that corresponds to the fatty acids $18: 1$ and $16: 0$, which was also found in $50 \%$ of SM and in $100 \%$ of PI. Several poly-unsaturated fatty acid variants were also observed. In conclusion, short chained and (poly-) unsaturated fatty acids were most abundant in the PLs present in autophagosomes, which corresponds to recent studies investigating fatty acids in autophagosomes from Drosophila and yeast (Laczko-Dobos et al, 2021; Schutter et al., 2020).

Autophagosomes are exceptional vesicles with explicit requirements regarding membrane curvature, mobility, and fusion ability. Short chained and (poly-) unsaturated fatty acids increase membrane fluidity and accordingly are supportive for membrane curvature. The PL composition in combination with distinct fatty acid characteristics determine shape and biophysical properties of a membrane and thus autophagosome function is crucially linked to a particular structural and quantitative PL pattern. This is highlighted by the finding that the PL composition is unaffected by the different autophagy conditions, albeit the treatments have significant effects on the rate of autophagosome formation (Appendix Figure S3).

Here, we present a powerful method to isolate native autophagosomes. The FACS-based approach resulted in the purification of intact vesicles, which enabled a profound autophagosome content profiling. We showed that proteasome inhibition modifies autophagic degradation leading to a switch towards a specific selection of cargoes that counteracts the cellular disturbance. Interestingly, nutrient deprivation, while leading to an enhanced autophagic flux, only marginally affects the types of degradation-prone proteins within autophagosomes, emphasizing that the obligation to enhance general protein turnover to recycle building blocks does not require focusing on particular cargoes. Besides protein analysis, isolation of autophagosomes additionally enabled the investigation of phospholipids in autophagosomes. The identified PL pattern was unaffected by different autophagy rates and exhibited a 
predominant content of short chained, unsaturated fatty acids, highlighting the relevance of a particular PL composition to support the structural and functional requirements of autophagic vesicles.

In summary, we introduce a potent isolation method for native unmanipulated autophagosomes, which paves the way to identify the impact of different autophagy conditions on autophagosome cargo and phospholipid profiles without the requirement of exhaustive cellular fractionation or genetic manipulation.

\section{Materials and Methods}

\section{Cell culture}

HeLa cells were cultivated in DMEM, supplemented with $10 \%$ fetal bovine serum, $1 \mathrm{mM}$ sodium pyruvate, and $1 \mathrm{x}$ antibiotic-antimycotic solution at $37^{\circ} \mathrm{C}$ in a $5 \% \mathrm{CO}_{2}$-humidified atmosphere. Cell identity was authenticated via STR analysis and cells were regularly tested negative for the presence of mycoplasma using the Venor GeM Mycoplasma PCR detection kit (Minerva Biolabs, 11-1050). Stock solutions of bafilomycin $A_{1}$ (Biozol, TRCB110000) and MG132 (Sigma Aldrich, P0042) were prepared in DMSO. For starvation, cells were treated with serum-free EBSS for $2 \mathrm{~h}$. For proteasome inhibition, cells were incubated with $10 \mu \mathrm{M}$ MG132 for $8 \mathrm{~h}$. Bafilomycin $A_{1}$ was added to each treatment $2 \mathrm{~h}$ before cells were collected.

\section{Autophagosome isolation}

At least $1 \times 10^{7}$ cells were collected using Trypsin/EDTA, centrifuged with $306 \mathrm{x}$ $g$ for $4 \mathrm{~min}$ and resuspended in PBS supplemented with EDTA-free protease inhibitor (Roche, 11873580001). Cell disruption was performed using a UP5OH ultrasonic processor (Hielscher) for $3 \times 2 \mathrm{~s}$ with an amplitude of $60 \%$. Lysates were centrifuged with $3.000 \times g$ for $10 \mathrm{~min}$ at $4{ }^{\circ} \mathrm{C}$, supernatants were collected and centrifuged with $150.000 \times g$ (at $\mathrm{r}_{\mathrm{av}}$ ) for $30 \mathrm{~min}$ at $4^{\circ} \mathrm{C}$ using an Optima ${ }^{\mathrm{TM}}$ MAX-XP Ultracentrifuge (Beckman Coulter; TLA120.2 rotor). Pellets were resuspended in PBS and $4 \mu \mathrm{g}$ of primary antibody were added for $30 \mathrm{~min}$, followed by addition of $12 \mu \mathrm{g}$ Cy5-conjugated secondary antibody for $1 \mathrm{~h}$. The samples were centrifuged with $150.000 \times g$ (at $r_{a v}$ ) for 30 min at $4{ }^{\circ} \mathrm{C}$ and pellets 
were resuspended in PBS. Autophagosome sortings were performed using a BD FACSAria III SORP (BD Biosciences) equipped with a $70 \mu \mathrm{m}$ nozzle and a 1.0 FSC neutral density filter. The autophagosome containing compartment was first established using an FSC/SSC plot on a logarithmic scale, followed by a doublet discrimination gate using SSC-A/W. Autophagosomes were defined as Cy5-positive events (640nm, BP 670/30), whose positivity was conducted according to the background given by an unstained as well as a secondary antibody-only negative control. Cy5-positive autophagosomes were sorted at minimum speed (flow rate < 3.0) maintaining less than 19.000 events per second. Analysis was performed using FlowJo v10.6.1 (BD Biosciences). Primary antibodies: MAP1LC3B (Novus, NB100-2220); GABARAP+GABARAPL1+GABARAPL2 (Abcam, ab109364). Secondary antibody: Cy5 anti-rabbit (ImmunoResearch, 711-175-152).

\section{Co-localization analysis}

Approximately $1 \times 10^{6}$ cells were prepared for FACS analysis as described above using $1 \mu \mathrm{g}$ primary and $3 \mu \mathrm{g}$ secondary antibody. Here, a fluorophoreconjugated primary antibody $(1 \mu \mathrm{g})$ was used in parallel. Autophagosomes were defined as Cy5-positive (640 nm, BP 670/30) as well as Alexa Fluor 488or Fluorescein-positive events (488 nm, BP 530/30). Positivity was conducted according to the background given by an unstained, a secondary antibody-only, and an unconjugated primary antibody/secondary antibody (Cy5)-only negative control. Analysis was performed using FlowJo v10.6.1 (BD Biosciences). Primary antibodies: MAP1LC3B (Novus, NB100-2220); GABARAP + GABARAPL1 + GABARAPL2 (Abcam, ab109364); MAP1LC3B (fluorescein labeled) (Enzo, BML-PW1205-0025); GABARAP Alexa Fluor 488 (SantaCruz, sc-377300 AF488). Secondary antibody: Cy5 anti-rabbit (ImmunoResearch, 711-175-152).

\section{Proteinase K digest}

$5 \mu \mathrm{g} / \mathrm{ml}$ proteinase $\mathrm{K}$ were supplemented to purified autophagosomes $\left(5 \times 10^{6}\right)$ and incubated for $15 \mathrm{~min}$ on ice. To stop enzyme activity, PMSF (100 mM) was added for $5 \mathrm{~min}$ on ice and samples were immediately precipitated using a 
methanol/chloroform (2:1) precipitation protocol. For positive control, isolated autophagosomes were sonicated using a UP50H ultrasonic processor (Hielscher) for $3 \times 3 \mathrm{~s}$ with an amplitude of $80 \%$ before proteinase $\mathrm{K}$ supplementation. For negative control, $5 \mu \mathrm{g} / \mathrm{ml}$ BSA instead of proteinase $\mathrm{K}$ were added to the samples.

\section{Western blotting}

Western blot analyses were performed as previously described (Bekbulat et al, 2020). Proteins of isolated autophagosomes were precipitated using a methanol/chloroform (2:1) precipitation protocol and resuspended in urea buffer (8 $\mathrm{M}$ urea and $4 \%(\mathrm{w} / \mathrm{v})$ CHAPS in $30 \mathrm{mM}$ Tris (pH 8.5 with $\mathrm{HCl}$ ), including EDTA-free protease inhibitor. Usually $5 \mu \mathrm{g}$ of total protein or the total protein of 1 to $2 \times 10^{6}$ autophagosomes were subjected to $4-12 \%$ NuPage Bis-Tris gels (Thermo Scientific, MP0335) and transferred onto a nitrocellulose membrane using the Trans-Blot Turbo RTA mini nitrocellulose transfer kit (BioRad, 1704270). After blocking with $5 \%$ fat-free milk in PBS-Tween 20, the membrane was probed with appropriate primary and HRP-conjugated secondary antibodies. Proteins were visualized via chemiluminescence using the Amersham Imager 600 (GE Healthcare Life Science). Quantification was performed using Aida Image Analyzer v4.26 (Raytest). Primary antibodies: MAP1LC3B (Sigma, L7543); GABARAP + GABARAPL1 + GABARAPL2 (Abcam, ab109364); SQSTM1 (Progen, GP62-C); Tubulin a (Sigma, T9026); Actin (Sigma, A5060). Secondary antibodies: Peroxidase anti-guinea pig (ImmunoResearch 706-035-148), Peroxidase anti-mouse (ImmunoResearch 715-035-151), Peroxidase anti-rabbit (ImmunoResearch 711-035-152).

\section{Immunocytochemistry}

Immunocytochemistry was conducted as previously described (Bekbulat et al., 2020). Briefly, cells were grown on glass cover slips, fixated using $4 \%$ PFA, and permeabilized with $90 \%$ (v/v) methanol. Unspecific binding sites were blocked with $3 \%$ BSA in PBS followed by incubation with primary antibody, fluorophore-conjugated secondary antibody as well as DAPI. Cells were imaged using the laser scanning microscope LSM710 (Zeiss, Germany). Primary 
antibody: MAP1LC3B (Nanotools, 0260-100). Secondary antibody: Cy3 antimouse (ImmunoResearch, 715-165-151).

\section{Differential interference contrast microscopy}

Isolated autophagosomes were concentrated using Amicon Ultra-15 filters (Merck, UFC901024) and dried on the surface of microscope slides. After sealing the samples with glass cover slips, they were imaged using the widefield microscope AF7000 (Leica).

\section{Negative stain electron microscopy}

$5 \mu \mathrm{l}$ of the FACS sorted autophagosome solution were applied onto freshly prepared Formvar coated copper mesh EM grids and incubated for 1 min at RT. Then, excess liquid was drained by using filter paper and grids were dried at RT. $10 \mu$ of a $2 \%(w / v)$ uranyl acetate (UA) solution was applied to the grid and incubated for $30 \mathrm{sec}$. The grid was washed $3 \times$ using drops of distilled water and drained using filter paper. Subsequently, the grid was dried over night at RT and analyzed using a TEM900 (Zeiss, Germany) operated at $80 \mathrm{keV}$ and equipped with a wide-angle dual-speed digital 2K CCD camera (TRS Tröndle).

\section{Sample preparation for LC-MS/MS proteomics}

$8 \times 10^{6}$ Cy5-positive autophagosomes were denatured with $2 \%$ sodium deoxycholate, $50 \mathrm{mM}$ Tris- $\mathrm{HCl} \mathrm{pH}$ 8.5, $2.5 \mathrm{mM}$ TCEP, $10 \mathrm{mM}$ chloroacetamide and protease inhibitor cocktail tablet (EDTA-free, Roche) at $95{ }^{\circ} \mathrm{C}$ for $10 \mathrm{~min}$. Lysates were prepared with in-StageTip (iST) processing method for LC-MS/MS as previously described by (Kulak et al, 2014). Briefly, proteins were digested overnight at $37^{\circ} \mathrm{C}$ with 1 vol. of $50 \mathrm{mM}$ Tris- $\mathrm{HCl} \mathrm{pH} 8.5$ containing LysC (Wako Chemicals) at $1: 100(\mathrm{w} / \mathrm{w})$ ratio and trypsin (Promega V5113) at 1:100 (w/w) ratio. Digestion was stopped with 2 vol. of $1 \%$ TFA in isopropanol. Digested peptides were purified with Empore SDB-RPS (styrenedivinylbenzene - reverse phase sulfonate) disks in stage tip (3M Empore) and were dried for LC-MS/MS.

\section{Mass spectrometry}


Dried peptides of each sample were resuspended in $2 \%(\mathrm{v} / \mathrm{v})$ acetonitrile / $1 \%$ (v/v) formic acid solution. Peptides were separated with Easy nLC 1200 (ThermoFisher Scientific) using a $30 \mathrm{~cm}$ long, $75 \mu \mathrm{m}$ inner diameter fused-silica column packed with $1.9 \mu \mathrm{m}$ C18 particles (ReproSil-Pur, Dr. Maisch) and kept at $50{ }^{\circ} \mathrm{C}$ using an integrated column oven (Sonation). Individual peptides were eluted by a non-linear gradient from 5 to $40 \%$ B over $120 \mathrm{~min}$, followed by a step-wise increase to $95 \%$ B in 6 min, which was kept for another 9 min and sprayed into a QExactive HF mass spectrometer (ThermoFisher Scientific). Full scan MS spectra $(300-1,650 \mathrm{~m} / \mathrm{z})$ were acquired with a resolution of 60.000 at $\mathrm{m} / \mathrm{z} 200$, maximum injection time of $20 \mathrm{~ms}$ and $A G C$ target value of $3 \times 10^{6}$. The 15 most intense precursors were selected for fragmentation (Top 15) and isolated with a quadrupole isolation window of 1.4 Th. MS2 scans were acquired in centroid mode with a resolution of 15,000 at $\mathrm{m} / \mathrm{z} 200$, a maximum injection time of $25 \mathrm{~ms}$, AGC target value of $1 \times 10^{5}$. lons were then fragmented using higher energy collisional dissociation (HCD) with a normalized collision energy (NCE) of 27; and the dynamic exclusion was set to $25 \mathrm{~s}$ to minimize the acquisition of fragment spectra of already acquired precursors.

\section{Processing of raw data and statistical analysis}

Raw files were analyzed with MaxQuant 1.6.17 with default settings using human trypsin digested "one sequence per gene" proteome database (Homo sapiens SwissProt database [TaxID:9606, version 2020-03-12]) with label free quantification (Cox \& Mann, 2008). Carbamidomethyl on cysteines were set as fixed modification and acetyl on the protein $\mathrm{N}$-term and methionine oxidation on methionines were set as variable modifications. Protein quantification and data normalization relied on the MaxLFQ algorithm implemented in MaxQuant and used for statistical analysis (Cox et al, 2014). Proteins only identified by a single modified peptide or matching to the reversed or contaminants databases were removed. Only well-quantified proteins and showing no missing value in any of the samples were used for statistical analysis. Significantly altered proteins were determined by a two-sided, unpaired Student's $t$-test ( $p$-value $<0.05$ ) adding minimum fold-change cut-off $(>=0.5)$ with GraphPad Prism 6 or 
Microsoft Excel 2016. No fold change could be calculated are indicated as not determinable (nd) in the volcano plots.

\section{Network analysis}

Protein pathway network analysis was performed with Cytoscape (Shannon et al, 2003) (version 3.8.2) in combination with StringApp, which is using STRING database (Doncheva et al, 2019). KEGG databases were used to obtain pathway enrichment (Kanehisa et al, 2010).

\section{Data availability}

The mass spectrometry proteomics data have been deposited to the ProteomeXchange Consortium via the PRIDE partner repository (Perez-Riverol et al, 2019) with the dataset identifier PXD024419 (Username: reviewer_pxd024419@ebi.ac.uk; Password: 18d5GdUA).

\section{Lipidomics}

Lipids were extracted from autophagosomes using a liquid-liquid extraction method previously described (Lerner et al, 2017). Briefly, autophagosome preparations were allowed to thaw at $4{ }^{\circ} \mathrm{C}$. $1000 \mu \mathrm{l}$ methyl tert buthyl ether (MTBE) /methanol (10:3, v/v) spiked $10 \mu$ of internal standard mixture for PLs and SMs and $250 \mu \mathrm{l} 0.1 \%$ formic acid containing $5 \mu \mathrm{m}$ THL/URB and $10 \mu \mathrm{g} / \mathrm{ml}$ BHT were added and samples were subjected to homogenization for $20 \mathrm{sec}$ at 6000 rpm with a Precellys 24 (Bertin Technologies, Montigny-le-Bretonneux, France), were vortexed for $30 \mathrm{sec}$ at $4^{\circ} \mathrm{C}$, and centrifuged for $10 \mathrm{~min}$ at 13000 rpm at $4{ }^{\circ} \mathrm{C}$. Then samples were allowed to freeze at $-20^{\circ} \mathrm{C}$ for $15 \mathrm{~min}$ and the upper organic phase containing lipids was recovered and dried under a gentle stream of nitrogen at $37^{\circ} \mathrm{C}$. The dried lipid extracts were re-solubilized in $90 \mu \mathrm{l}$ methanol and stored at $-20{ }^{\circ} \mathrm{C}$ until analysis. The lower, aqueous phase containing autophagosomal proteins was stored at $-20^{\circ} \mathrm{C}$ for further BCA assay protein content determination, which was used for normalization of PL values. Lipids were analyzed by liquid-chromatography multiple reaction monitoring (LC/MRM) on a QTRAP 55500 mass spectrometer (AB Sciex, Darmstadt, Germany) operated in positive negative ion mode switching. The 
chromatographic, ionization and detection conditions were set as previously described (Lerner et al., 2017). The MRM transitions for the analysis of selected PLs and SMs as well as calibration standards used for quantification were set as previously reported (Lerner et al, 2019; Lerner et al., 2017). For LC/MRM analysis, $3 \mu \mathrm{l}$ water were added to a $27 \mu$ methanolic solution of extracted lipids and $10 \mu \mathrm{l}$ of this solution were injected into the LC/MRM instrument. Lipids were quantified using Multiquant 3.0.3 software and determined lipid levels were normalized to the protein content of the autophagosomes.

\section{Statistics}

In dependence of normal distribution or variance differences, statistical significance between the samples were determined by unpaired Student's $t$-test or One-Way ANOVA using SIGMA STAT (SPSS Science). The results are shown as mean \pm standard deviation (SD). Statistical significance was accepted at a level of $p<0.05$ ( $p$ value $\leq 0.05={ }^{*}, \leq 0.01=^{* *}, \leq 0.001=^{* * *}$ ).

\section{Acknowledgments}

We would like to thank Drs Stefanie Möckel and Jesús Gil-Pulido of the Flow Cytometry Core facility as well as Drs Sandra Ritz and Petri Turunen of the Microscopy and Histology Core facility, both Institute of Molecular Biology, Mainz, for scientific help and discussions. In addition, we thank Marion Basoglu for help with EM experiments conducted in the FB15 EM Facility of the Goethe University Frankfurt, which is part of the Frankfurt Center for Electron Microscopy (FCEM). This work was funded by the Deutsche Forschungsgemeinschaft (DFG, German Research Foundation) - Project-ID 259130777-SFB1177. Lipidomic experiments were financially supported by the Core Facility of the Clinical Lipidomics Unit.

\section{Author contributions}

D.S. was supported by P.H.D. and H.H. and performed all the experiments, except TEM, conducted by S.E., proteomics, conducted by S.B., G.T., and C.M., and lipidomics, conducted by L.B. All authors participated in data analysis 
and discussion. D.S., C.B., and A.K. conceived the study and wrote the manuscript.

\section{Conflict of interest}

The authors declare no conflict of interest.

\section{References}

Arndt V, Dick N, Tawo R, Dreiseidler M, Wenzel D, Hesse M, Furst DO, Saftig $P$, Saint R, Fleischmann BK et al (2010) Chaperone-assisted selective autophagy is essential for muscle maintenance. Curr Biol 20: 143-148

Bekbulat F, Schmitt D, Feldmann A, Huesmann H, Eimer S, Juretschke T, Beli P, Behl C, Kern A (2020) RAB18 Loss Interferes With Lipid Droplet Catabolism and Provokes Autophagy Network Adaptations. J Mol Biol 432: 1216-1234

Condon KJ, Sabatini DM (2019) Nutrient regulation of mTORC1 at a glance. $J$ Cell Sci 132

Cox J, Hein MY, Luber CA, Paron I, Nagaraj N, Mann M (2014) Accurate proteome-wide label-free quantification by delayed normalization and maximal peptide ratio extraction, termed MaxLFQ. Mol Cell Proteomics 13: 2513-2526

Cox J, Mann M (2008) MaxQuant enables high peptide identification rates, individualized p.p.b.-range mass accuracies and proteome-wide protein quantification. Nat Biotechnol 26: 1367-1372

de la Ballina LR, Munson MJ, Simonsen A (2020) Lipids and Lipid-Binding Proteins in Selective Autophagy. J Mol Biol 432: 135-159

De Tito S, Hervas JH, van Vliet AR, Tooze SA (2020) The Golgi as an Assembly Line to the Autophagosome. Trends Biochem Sci 45: 484-496

Dengjel J, Hoyer-Hansen M, Nielsen MO, Eisenberg T, Harder LM, Schandorff S, Farkas T, Kirkegaard T, Becker AC, Schroeder S et al (2012) Identification of autophagosome-associated proteins and regulators by quantitative proteomic analysis and genetic screens. Mol Cell Proteomics 11: M111 014035

Doncheva NT, Morris JH, Gorodkin J, Jensen LJ (2019) Cytoscape StringApp: Network Analysis and Visualization of Proteomics Data. J Proteome Res 18: 623-632 
Gamerdinger M, Hajieva P, Kaya AM, Wolfrum U, Hartl FU, Behl C (2009) Protein quality control during aging involves recruitment of the macroautophagy pathway by BAG3. EMBO J 28: 889-901

Gao W, Kang JH, Liao Y, Ding WX, Gambotto AA, Watkins SC, Liu YJ, Stolz DB, Yin XM (2010) Biochemical isolation and characterization of the tubulovesicular LC3-positive autophagosomal compartment. J Biol Chem 285: 1371-1383

Gatica D, Lahiri V, Klionsky DJ (2018) Cargo recognition and degradation by selective autophagy. Nat Cell Biol 20: 233-242

Gewaid H, Aoyagi H, Arita M, Watashi K, Suzuki R, Sakai S, Kumagai K, Yamaji T, Fukasawa M, Kato F et al (2020) Sphingomyelin Is Essential for the Structure and Function of the Double-Membrane Vesicles in Hepatitis C Virus RNA Replication Factories. J Virol 94

He C, Klionsky DJ (2009) Regulation mechanisms and signaling pathways of autophagy. Annu Rev Genet 43: 67-93

Kabeya Y, Mizushima N, Yamamoto A, Oshitani-Okamoto S, Ohsumi Y, Yoshimori T (2004) LC3, GABARAP and GATE16 localize to autophagosomal membrane depending on form-II formation. J Cell Sci 117: 2805-2812

Kanehisa M, Goto S, Furumichi M, Tanabe M, Hirakawa M (2010) KEGG for representation and analysis of molecular networks involving diseases and drugs. Nucleic Acids Res 38: D355-360

Khaminets A, Behl C, Dikic I (2016) Ubiquitin-Dependent And Independent Signals In Selective Autophagy. Trends Cell Biol 26: 6-16

Kirkin V, McEwan DG, Novak I, Dikic I (2009) A role for ubiquitin in selective autophagy. Mol Cell 34: 259-269

Kulak NA, Pichler G, Paron I, Nagaraj N, Mann M (2014) Minimal, encapsulated proteomic-sample processing applied to copy-number estimation in eukaryotic cells. Nat Methods 11: 319-324

Laczko-Dobos H, Maddali AK, Jipa A, Bhattacharjee A, Vegh AG, Juhasz G (2021) Lipid profiles of autophagic structures isolated from wild type and Atg2 mutant Drosophila. Biochim Biophys Acta Mol Cell Biol Lipids 1866: 158868

Lamb CA, Yoshimori T, Tooze SA (2013) The autophagosome: origins unknown, biogenesis complex. Nat Rev Mol Cell Biol 14: 759-774 
Le Guerroue F, Eck F, Jung J, Starzetz T, Mittelbronn M, Kaulich M, Behrends C (2017) Autophagosomal Content Profiling Reveals an LC3C-Dependent Piecemeal Mitophagy Pathway. Mol Cell 68: 786-796 e786

Lerner R, Pascual Cuadrado D, Post JM, Lutz B, Bindila L (2019) Broad Lipidomic and Transcriptional Changes of Prophylactic PEA Administration in Adult Mice. Front Neurosci 13: 527

Lerner R, Post J, Loch S, Lutz B, Bindila L (2017) Targeting brain and peripheral plasticity of the lipidome in acute kainic acid-induced epileptic seizures in mice via quantitative mass spectrometry. Biochim Biophys Acta Mol Cell Biol Lipids 1862: 255-267

Mancias JD, Wang X, Gygi SP, Harper JW, Kimmelman AC (2014) Quantitative proteomics identifies NCOA4 as the cargo receptor mediating ferritinophagy. Nature 509: 105-109

Mercer TJ, Gubas A, Tooze SA (2018) A molecular perspective of mammalian autophagosome biogenesis. J Biol Chem 293: 5386-5395

Mizushima N (2020) The ATG conjugation systems in autophagy. Curr Opin Cell Biol 63: 1-10

Mizushima N, Komatsu M (2011) Autophagy: renovation of cells and tissues. Cell 147: 728-741

Mizushima N, Ohsumi Y, Yoshimori T (2002) Autophagosome formation in mammalian cells. Cell Struct Funct 27: 421-429

Nascimbeni AC, Giordano F, Dupont N, Grasso D, Vaccaro MI, Codogno P, Morel E (2017) ER-plasma membrane contact sites contribute to autophagosome biogenesis by regulation of local PI3P synthesis. EMBO J 36: 2018-2033

Nguyen TN, Padman BS, Usher J, Oorschot V, Ramm G, Lazarou M (2016) Atg8 family LC3/GABARAP proteins are crucial for autophagosome-lysosome fusion but not autophagosome formation during PINK1/Parkin mitophagy and starvation. J Cell Biol 215: 857-874

Noda NN, Inagaki F (2015) Mechanisms of Autophagy. Annu Rev Biophys 44: 101-122

Osawa T, Ishii Y, Noda NN (2020) Human ATG2B possesses a lipid transfer activity which is accelerated by negatively charged lipids and WIPI4. Genes Cells 25: 65-70 
Osawa T, Kotani T, Kawaoka T, Hirata E, Suzuki K, Nakatogawa H, Ohsumi Y, Noda NN (2019) Atg2 mediates direct lipid transfer between membranes for autophagosome formation. Nat Struct Mol Biol 26: 281-288

Pankiv S, Clausen TH, Lamark T, Brech A, Bruun JA, Outzen H, Overvatn A, Bjorkoy G, Johansen T (2007) p62/SQSTM1 binds directly to Atg8/LC3 to facilitate degradation of ubiquitinated protein aggregates by autophagy. $\mathrm{J}$ Biol Chem 282: 24131-24145

Perez-Riverol Y, Csordas A, Bai J, Bernal-Llinares M, Hewapathirana S, Kundu DJ, Inuganti A, Griss J, Mayer G, Eisenacher M et al (2019) The PRIDE database and related tools and resources in 2019: improving support for quantification data. Nucleic Acids Res 47: D442-D450

Pohl C, Dikic I (2019) Cellular quality control by the ubiquitin-proteasome system and autophagy. Science 366: 818-822

Sanjuan MA, Dillon CP, Tait SW, Moshiach S, Dorsey F, Connell S, Komatsu M, Tanaka K, Cleveland JL, Withoff S et al (2007) Toll-like receptor signalling in macrophages links the autophagy pathway to phagocytosis. Nature 450: 12531257

Schutter M, Giavalisco P, Brodesser S, Graef M (2020) Local Fatty Acid Channeling into Phospholipid Synthesis Drives Phagophore Expansion during Autophagy. Cell 180: 135-149 e114

Shannon P, Markiel A, Ozier O, Baliga NS, Wang JT, Ramage D, Amin N, Schwikowski B, Ideker T (2003) Cytoscape: a software environment for integrated models of biomolecular interaction networks. Genome Res 13: 24982504

Slobodkin MR, Elazar Z (2013) The Atg8 family: multifunctional ubiquitin-like key regulators of autophagy. Essays Biochem 55: 51-64

Slotte JP (2013) Biological functions of sphingomyelins. Prog Lipid Res 52: 424437

Stolz A, Ernst A, Dikic I (2014) Cargo recognition and trafficking in selective autophagy. Nat Cell Biol 16: 495-501

Tsuboyama K, Koyama-Honda I, Sakamaki Y, Koike M, Morishita H, Mizushima N (2016) The ATG conjugation systems are important for degradation of the inner autophagosomal membrane. Science 354: 1036-1041 
Valverde DP, Yu S, Boggavarapu V, Kumar N, Lees JA, Walz T, Reinisch KM, Melia TJ (2019) ATG2 transports lipids to promote autophagosome biogenesis. J Cell Biol 218: 1787-1798

Velikkakath AK, Nishimura T, Oita E, Ishihara N, Mizushima N (2012) Mammalian Atg2 proteins are essential for autophagosome formation and important for regulation of size and distribution of lipid droplets. Mol Biol Cell 23: 896-909

Weidberg H, Shvets E, Shpilka T, Shimron F, Shinder V, Elazar Z (2010) LC3 and GATE-16/GABARAP subfamilies are both essential yet act differently in autophagosome biogenesis. EMBO J 29: 1792-1802

Zellner S, Schifferer M, Behrends C (2021) Systematically defining selective autophagy receptor-specific cargo using autophagosome content profiling. Mol Cell

\section{Figure legends}

Figure 1. FACS-based isolation of autophagosomes.

A. Schematic representation of the FACS-based autophagosome isolation method. TL total lysate; P pellet fraction; S supernatant.

B. Western blotting of different numbers of purified autophagosomes. Isolations were based on LC3B or all GABARAP antibodies, respectively, and are represented with total lysate (TL). Depicted are representative blots of 12 independent approaches.

C. Co-localization of fluorescence signals linked to LC3B and GABARAP. Shown percentages represent the average distribution of 3 independent experiments excluding double negative events.

D. Co-localization of fluorescence signals linked to LC3B and all GABARAP isoforms. Shown percentages represent the average distribution of 3 independent experiments excluding double negative events.

$\mathrm{C}+\mathrm{D}$. Statistics are depicted as mean $\pm \mathrm{SD}$; One-Way ANOVA; ${ }^{*} \mathrm{p} \leq 0.05$; ${ }^{* *} \mathrm{p} \leq$ $0.01 ;{ }^{* * *} p \leq 0.001$.

Figure 2. FACS-based isolated autophagosomes are sealed. 
A. Western blotting of isolated autophagosomes upon proteinase $\mathrm{K}$ digestion. Mechanically opened vesicles served as positive control. For negative control, isolates were incubated with BSA instead of proteinase K. Depicted are two different blots that are representative for 5 independent experiments.

B. DIC microscopy images of purified autophagosomes at high (I) or low (II) dilution. Images are representative for 3 independent approaches. Scale bar $=$ $10 \mu \mathrm{m}$.

C. Negative stain electron microscopy images of isolated autophagosomes. Scale bar $=500 \mathrm{~nm}$.

D. Size evaluation of isolated autophagosomes. The diameter of approx. 60 individual autophagosomes was determined using EM images. Statistics are depicted as mean \pm SD.

\section{Figure 3. Protein cargo profiles of isolated autophagosomes upon different autophagy conditions.}

A. KEGG pathway analysis of top 500 basal cargo proteins. Pathways are shown with the number of proteins found in the data set and computed FDRs for enrichment. Represented are the top 10 pathways.

B. Volcano plot showing the differential appearance of proteins in autophagosomes of EBSS treated cells in comparison to control cells kept in full medium. $\log _{2}$-transformed fold changes. For proteins that were excluded from autophagosomes upon EBSS treatment or which exclusively appeared within these vesicles, no fold changes could be calculated and are indicated as not determinable (nd).

C. Volcano plot showing the differential appearance of proteins in autophagosomes of MG132 treated cells in comparison to control cells kept in full medium. Log $_{2}$-transformed fold changes. For proteins that were excluded from autophagosomes upon MG132 treatment or which exclusively appeared within these vesicles, no fold changes could be calculated and are indicated as not determinable (nd).

D. KEGG pathway analysis of proteins with enhanced autophagosome levels upon MG132 treatment. Pathways are presented with the number of proteins found in in the data set and computed FDRs for enrichment. 
E. KEGG pathway analysis of proteins with reduced autophagosome levels upon MG132 treatment. Pathways are presented with the number of proteins found in in the data set and computed FDRs for enrichment. Represented are the top 3 pathways. See Appendix Figure S7 for complete list.

Figure 4. Phospholipid profiles of isolated autophagosomes upon different autophagy conditions.

A. Relative phospholipid composition of isolated autophagosomes upon different autophagy conditions. PL ratios were calculated based on their concentration determined in the samples. PC phosphatidylcholine, LPC lysophosphatidylcholine, SM sphingomyelin, PS phosphatidylserine, PE phosphatidylethanolamine, PI phosphatidylinositol, LPE lysophosphatidylethanolamine, PG phosphatidylglycerol.

B. Relative fatty acid combinations of identified phospholipids upon different autophagy conditions. Fatty acid ratios were calculated based on their concentration measured in the samples.

$A+B$. Statistics are depicted as mean $\pm S D$; statistical analysis resulted in no significant differences between the different treatments (One-Way ANOVA, $n=$ 3). 
A

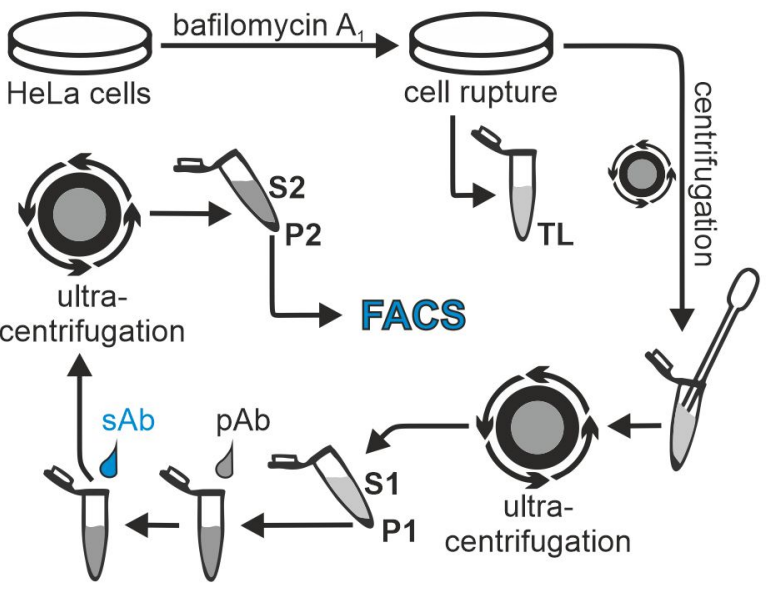

B

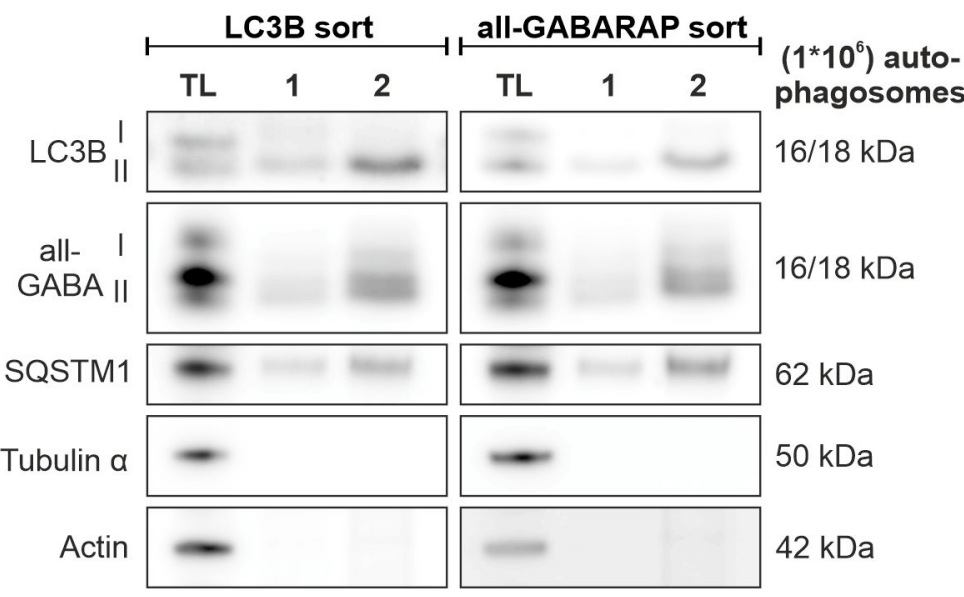

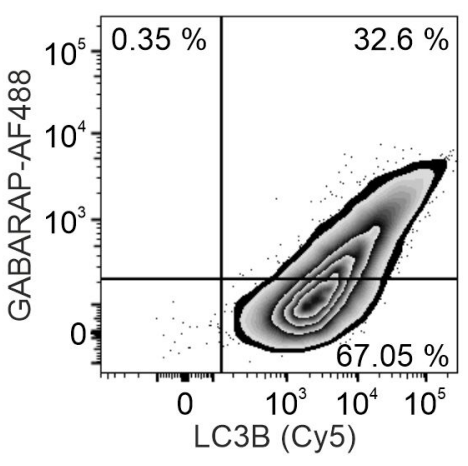

듬

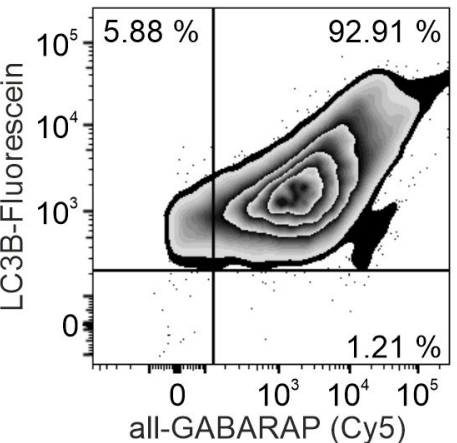

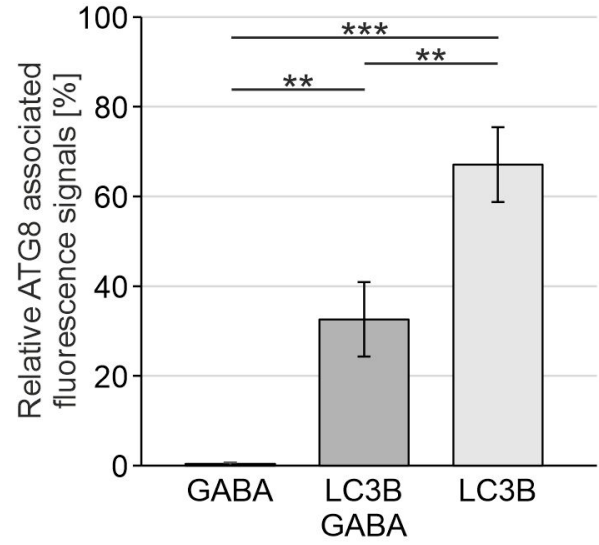

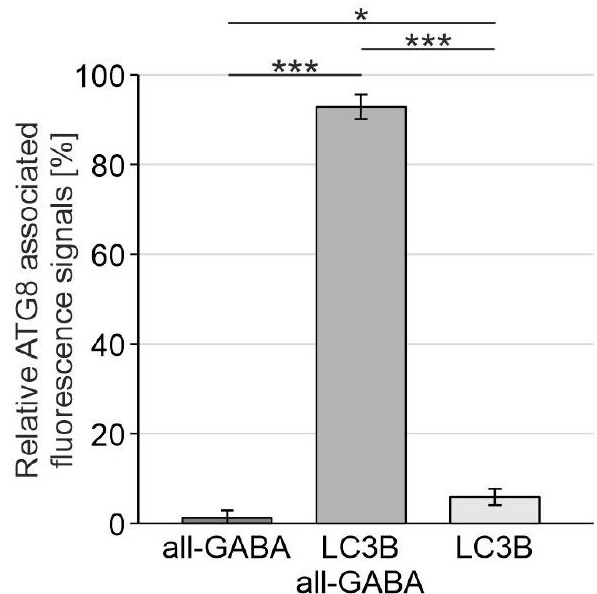


A
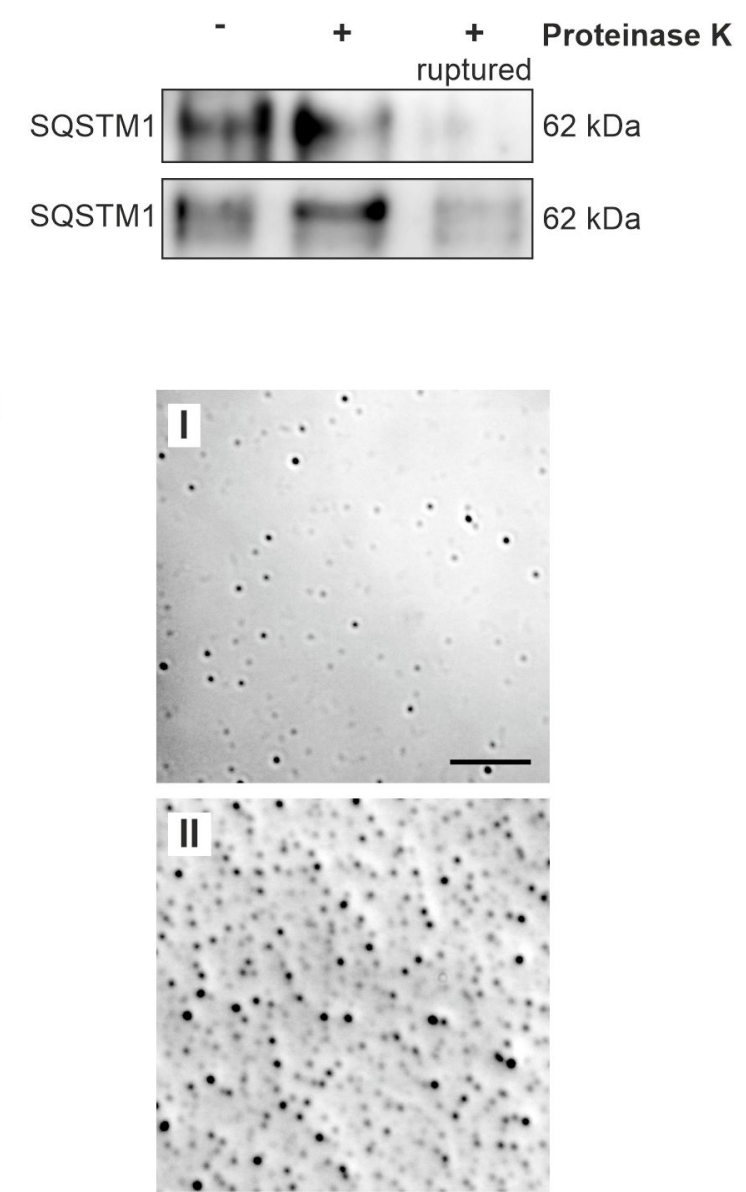

C
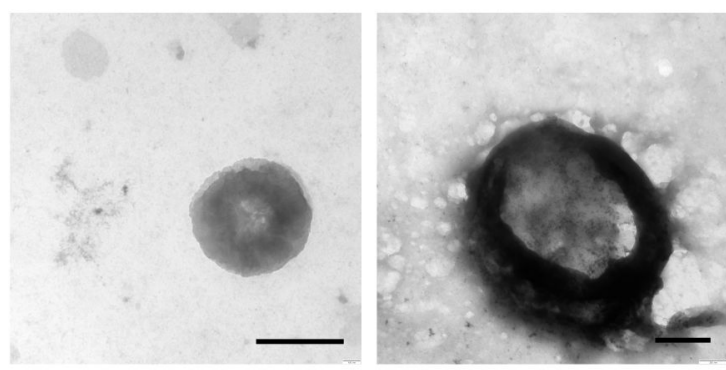

D

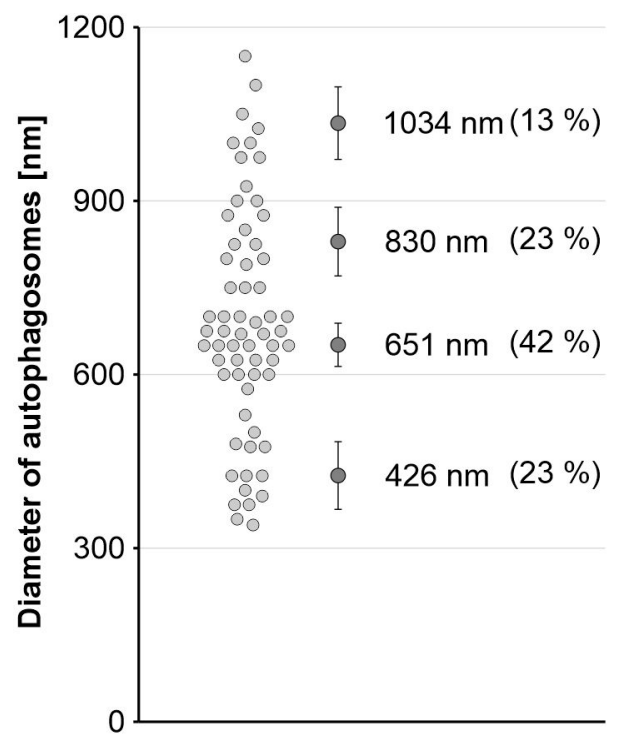




\begin{tabular}{|l|l|l|l|}
\hline ID & Pathway & Count & FDR \\
\hline hsa03010 & Ribosome & 68 & $1.39 \mathrm{e}-56$ \\
\hline hsa03040 & Spliceosome & 37 & $1.24 \mathrm{e}-22$ \\
\hline hsa04141 & Protein processing in endoplasmic reticulum & 35 & $2.88 \mathrm{e}-18$ \\
\hline hsa04145 & Phagosome & 21 & $6.43 \mathrm{e}-08$ \\
\hline hsa05016 & Huntington's disease & 23 & $2.49 \mathrm{e}-07$ \\
\hline hsa05012 & Parkinson's disease & 19 & $8.52 \mathrm{e}-07$ \\
\hline hsa03060 & Protein export & 9 & $2.01 \mathrm{e}-06$ \\
\hline hsa04144 & Endocytosis & 23 & $7.01 \mathrm{e}-06$ \\
\hline hsa04612 & Antigen processing and presentation & 12 & $1.31 \mathrm{e}-05$ \\
\hline hsa04260 & Cardiac muscle contraction & 12 & $4.46 \mathrm{e}-05$ \\
\hline
\end{tabular}

B

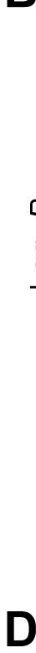

C

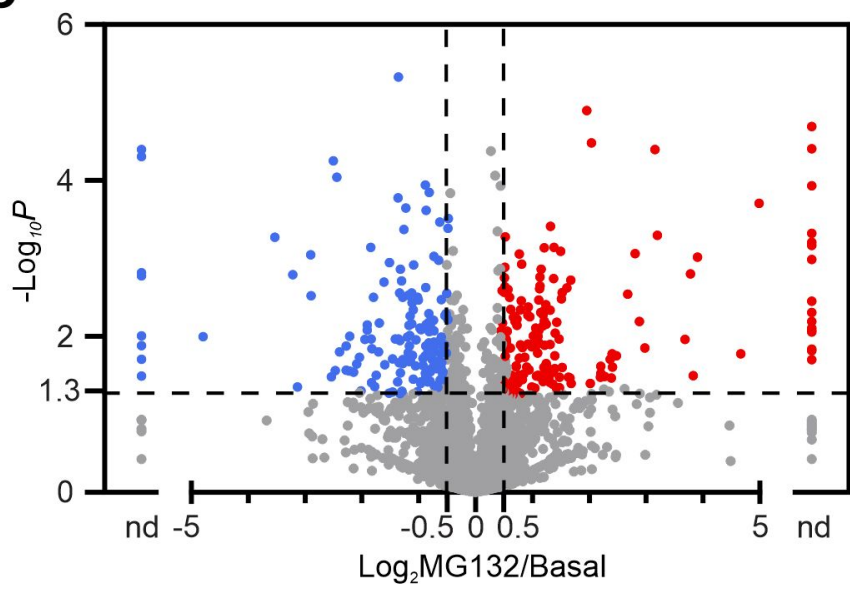

\begin{tabular}{|l|l|l|l|}
\hline ID & Pathway & Count & FDR \\
\hline hsa03050 & Proteasome & 33 & $4.59 \mathrm{e}-47$ \\
\hline hsa05169 & Epstein-Barr virus infection & 20 & $1.22 \mathrm{e}-13$ \\
\hline hsa04141 & Protein processing in endoplasmic reticulum & 12 & $1.33 \mathrm{e}-06$ \\
\hline
\end{tabular}

\begin{tabular}{|l|l|l|l|}
\hline ID & Pathway & Count & FDR \\
\hline hsa04142 & Lysosome & 30 & $3.02 \mathrm{e}-32$ \\
\hline hsa00511 & Other glycan degradation & 8 & $6.13 \mathrm{e}-10$ \\
\hline hsa00531 & Glycosaminoglycan degradation & 8 & $6.13 \mathrm{e}-10$ \\
\hline
\end{tabular}


bioRxiv preprint doi: https://doi.org/10.1101/2021.04.16.440117; this version posted April 17, 2021. The copyright holder for this preprint (which was not certified by peer review) is the author/funder. All rights reserved. No reuse allowed without permission.

A
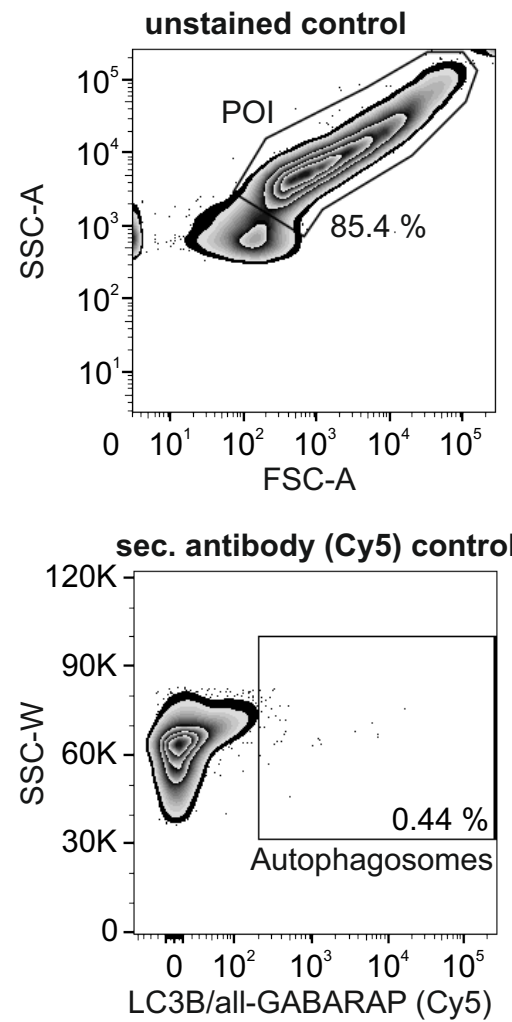
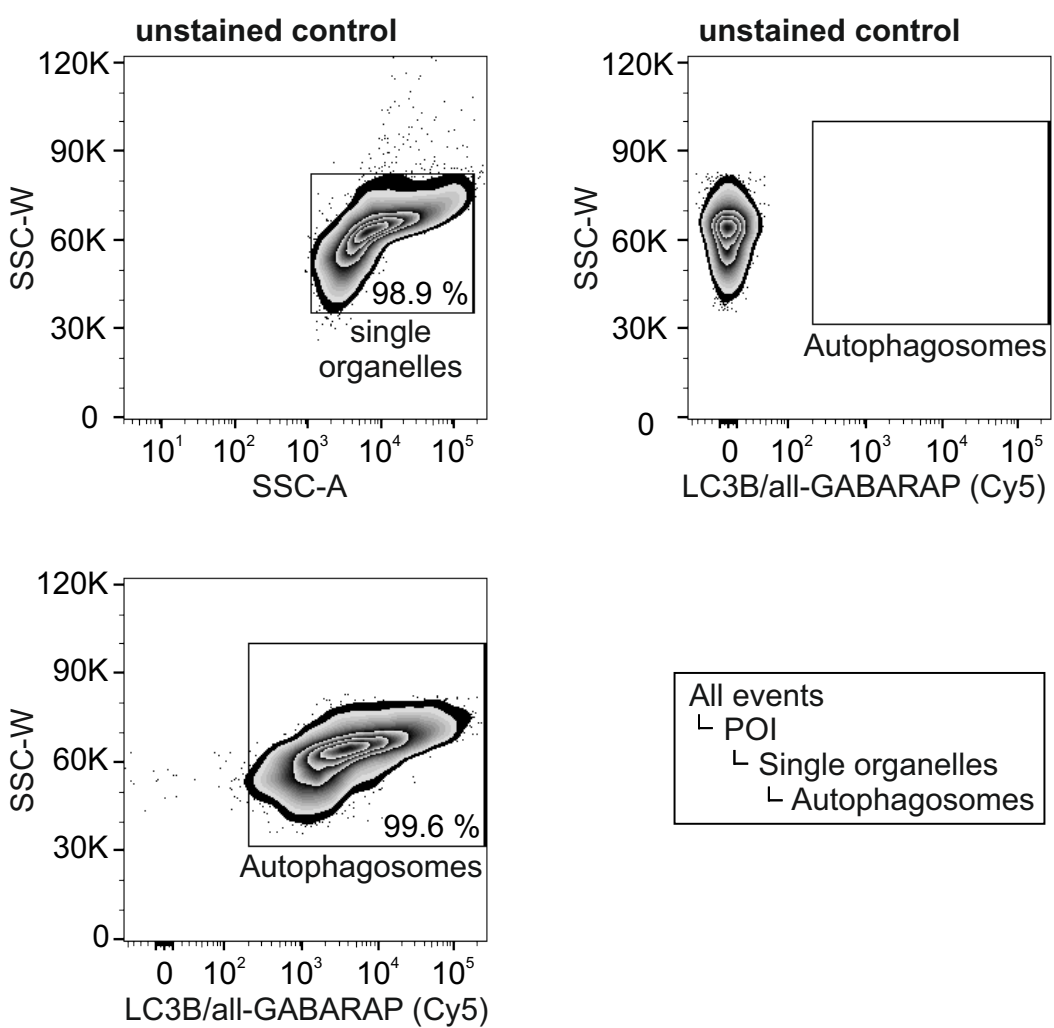

$$
\begin{aligned}
& \text { All events } \\
& \text { L POI } \\
& \text { L Single organelles } \\
& \text { L Autophagosomes }
\end{aligned}
$$

B

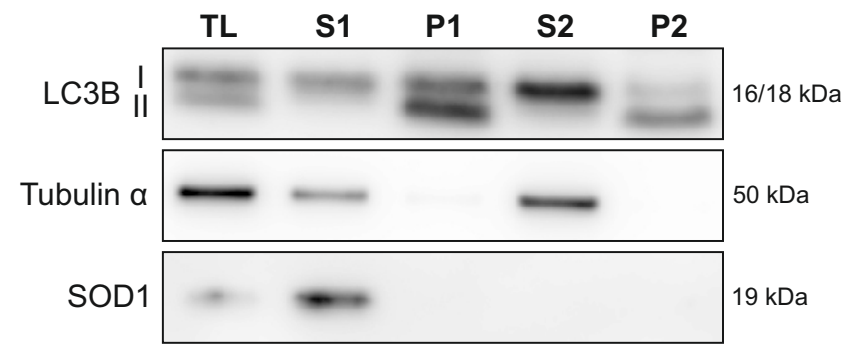

\section{Appendix Figure S1. FACS-based autophagosome isolation.}

A. Representative images of the FACS gating strategy. The population of interest (POI) containing autophagosomes was first established using a FSC/SSC plot on a logarithmic scale, followed by a doublet discrimination gate using SSC-A/W. Cy5-positive events (640nm, BP 670/30) were defined as autophagosomes, whose positivity was conducted according to the background given by an unstained as well as a secondary antibody-only negative control.

B. Western blot analysis of different steps of the isolation protocol. TL total lysate, S supernatant, P pellet. Shown is a representative blot of 3 independent approaches. 

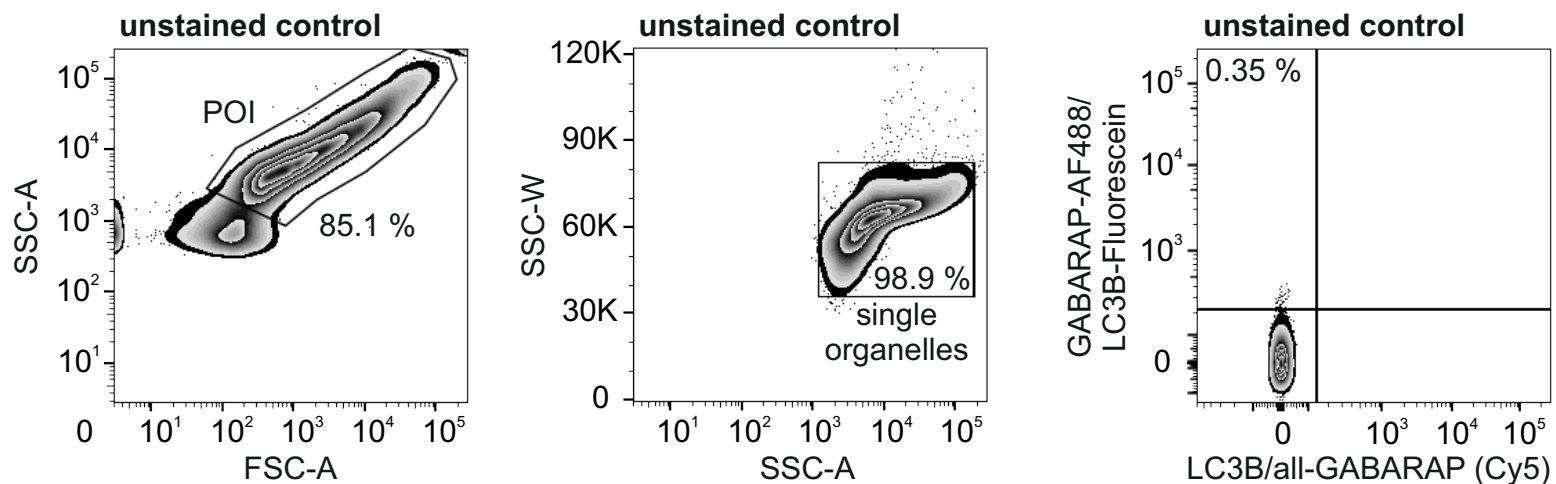

sec. antibody (Cy5) control

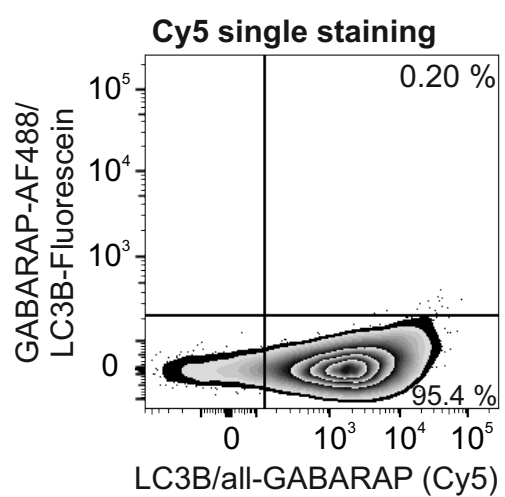

\section{Appendix Figure S2. Gating strategy of the co-localization assay.}

The population of interest (POI) containing autophagosomes was first established using a FSC/SSC plot on a logarithmic scale, followed by a doublet discrimination gate using SSC-A/W. Autophagosomes were defined as Cy5-positive events (640 nm, BP 670/30) and Alexa Fluor 488- or Fluorescein-positive events (488 nm, BP 530/30). Their positivity was conducted according to the background given by an unstained, a secondary antibody (Cy5)-only as well as a primary antibody/secondary antibody (Cy5)-only negative control. 
A
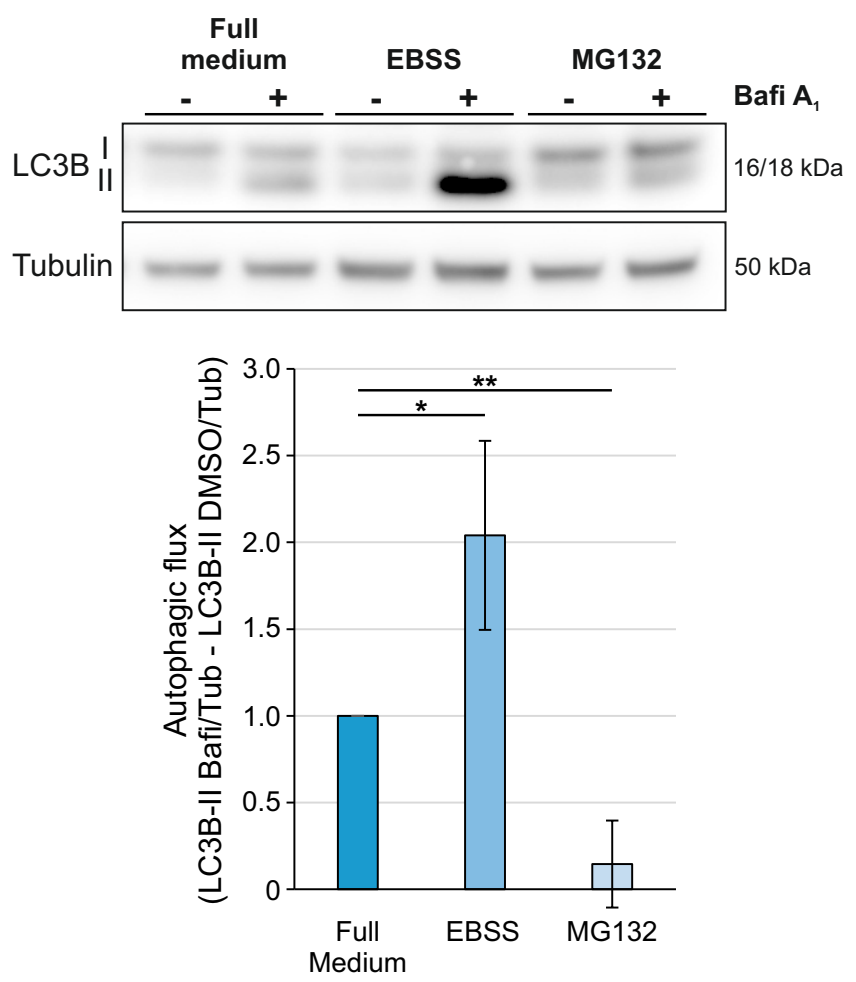

B
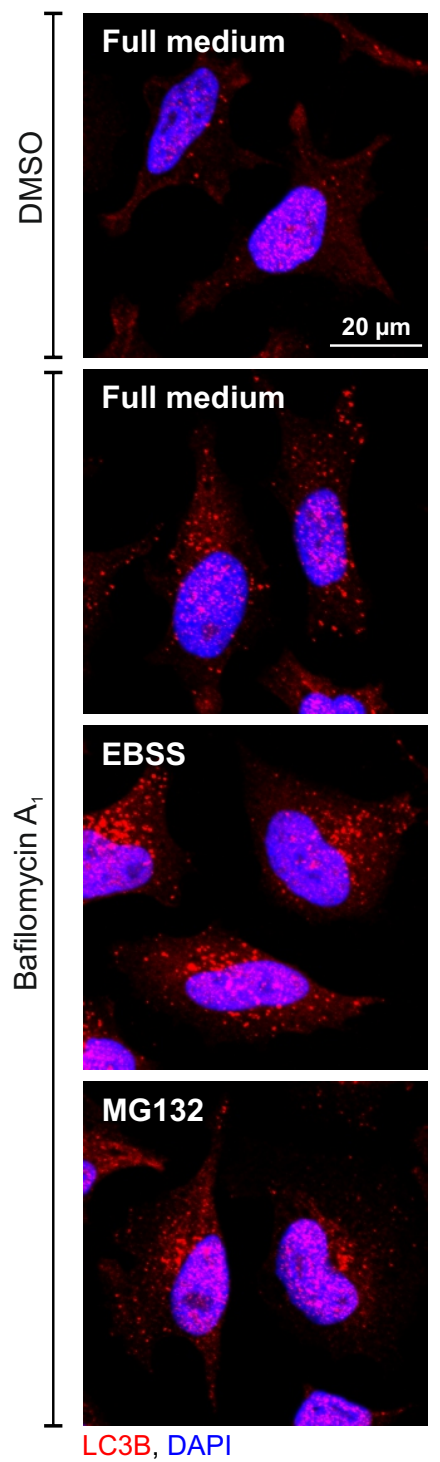

\section{Appendix Figure S3. Effects of different treatments on autophagy.}

A. Western blot analysis of autophagic activity (LC3B-II flux) upon different treatment paradigms. Cells were treated with DMSO or bafilomycin $A_{1}$. LC3B-Il levels were corrected over the loading control tubulin. Statistics are depicted as mean $\pm S D$. $\left(n=3,{ }^{*} p \leq 0.05\right.$; $\left.{ }^{* *} p \leq 0.01\right)$.

B. Representative confocal images of immunocytochemical stainings of LC3B (red) upon different treatment paradigms. Nuclei were stained with DAPI (blue). 


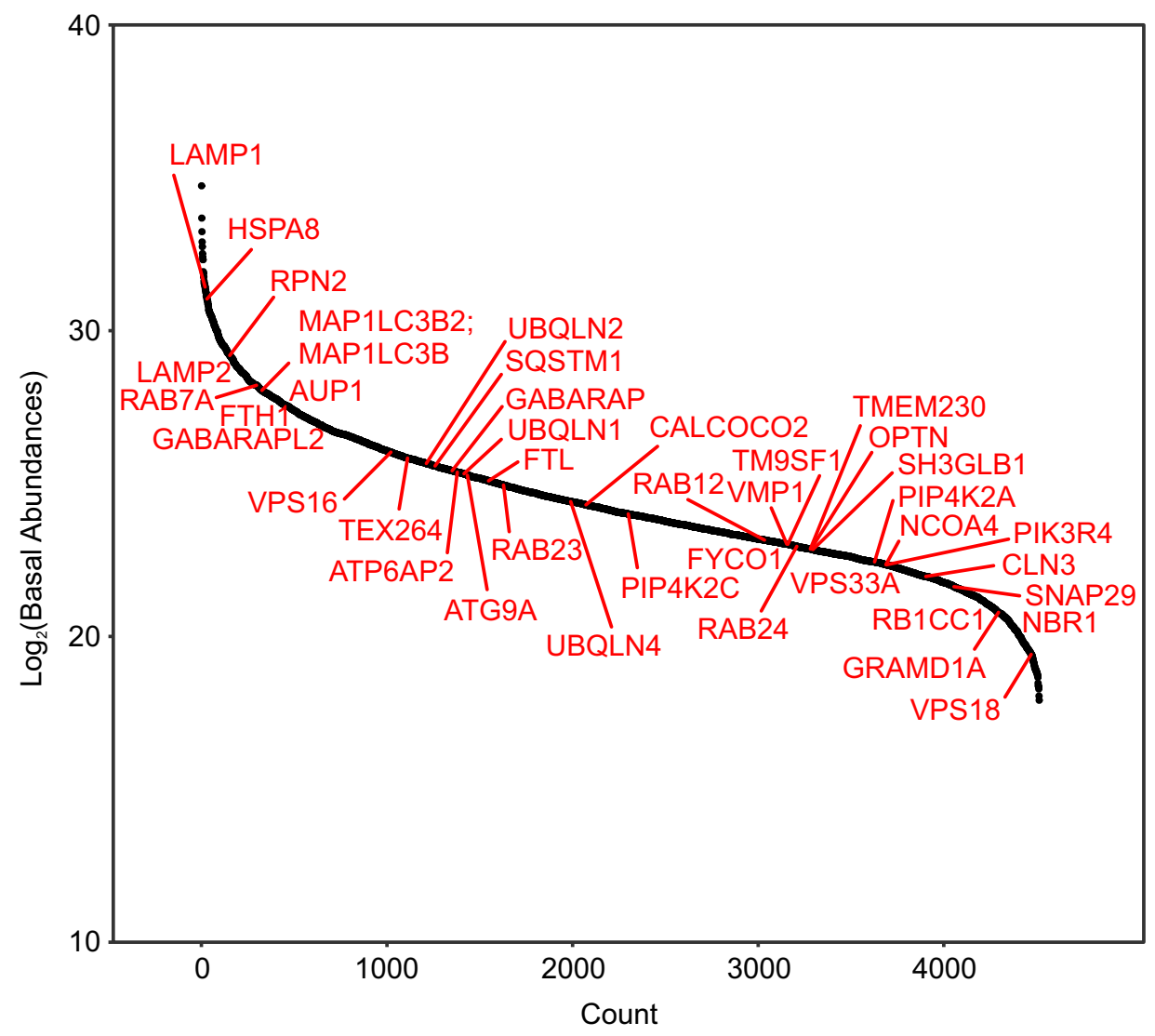

\section{Appendix Figure S4. Autophagosomal proteins in basal autophagosomes.}

Abundance plot of autophagosome proteins detected in isolated autophagosomes upon basal conditions. 
bioRxiv preprint doi: https://doi.org/10.1101/2021.04.16.440117; this version posted April 17, 2021. The copyright holder for this preprint (which was not certified by peer review) is the author/funder. All rights reserved. No reuse allowed without permission.

A

\begin{tabular}{|l|l|l|l|l|}
\hline ID & Pathway & Count_N1 & Count_N2 & Count_N3 \\
\hline hsa03010 & Ribosome & 67 & 66 & 66 \\
\hline hsa03040 & Spliceosome & 37 & 37 & 31 \\
\hline hsa04141 & Protein processing in endoplasmic reticulum & 36 & 36 & 36 \\
\hline hsa04145 & Phagosome & 21 & 22 & 21 \\
\hline hsa05016 & Huntington's disease & 22 & 24 & 24 \\
\hline hsa05012 & Parkinson's disease & 18 & 22 & 17 \\
\hline hsa03060 & Protein export & 11 & 8 & 10 \\
\hline hsa04144 & Endocytosis & 23 & 23 & 22 \\
\hline hsa04612 & Antigen processing and presentation & 12 & 12 & 12 \\
\hline hsa04260 & Cardiac muscle contraction & 12 & 12 & 11 \\
\hline
\end{tabular}

B

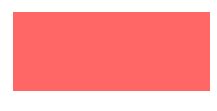

Ribosome

Biogenesis
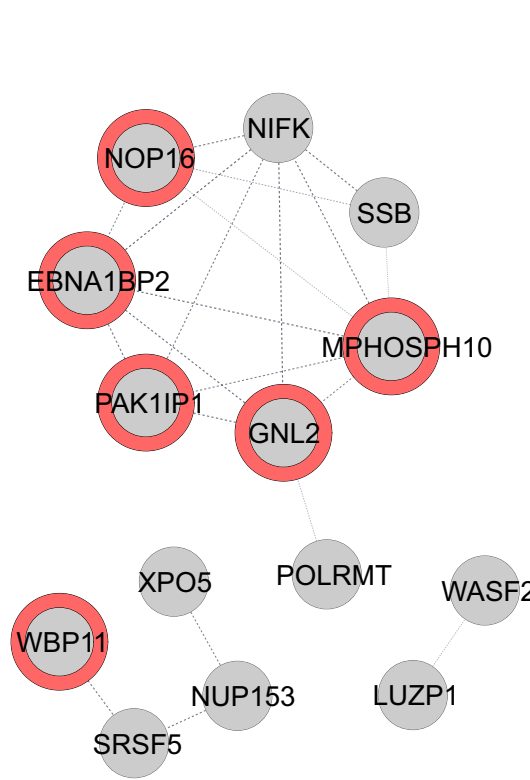

C

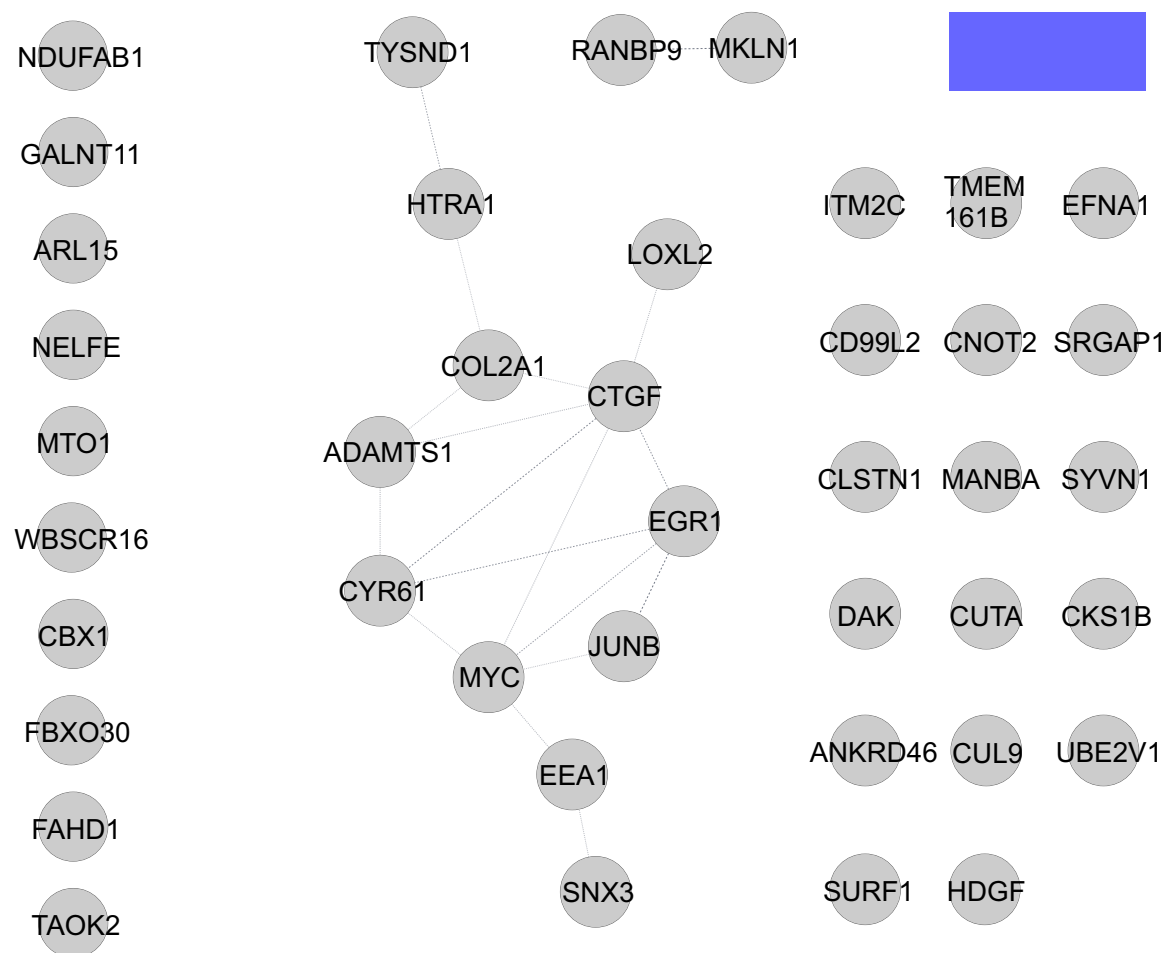

\section{Appendix Figure S5. Cargo analysis of autophagosomes upon basal and starved conditions.}

A. KEGG pathways are presented with the number of proteins found in the data set of each autophagosome analysis from 3 independent autophagosome isolations upon basal autophagy conditions.

B. String analysis of proteins with enhanced abundance in autophagosomes upon EBSS treatment.

C. String analysis of proteins with reduced abundance in autophagosomes upon EBSS treatment. 
AoRxiv preprint doi: https://doi.org/10.1101/2021.04.16.440117; this version posted April 17, 2021. The copyright holder for this preprint (which was not certified by peer review) is the author/funder. All rights reserved. No reuse allowed without permission.

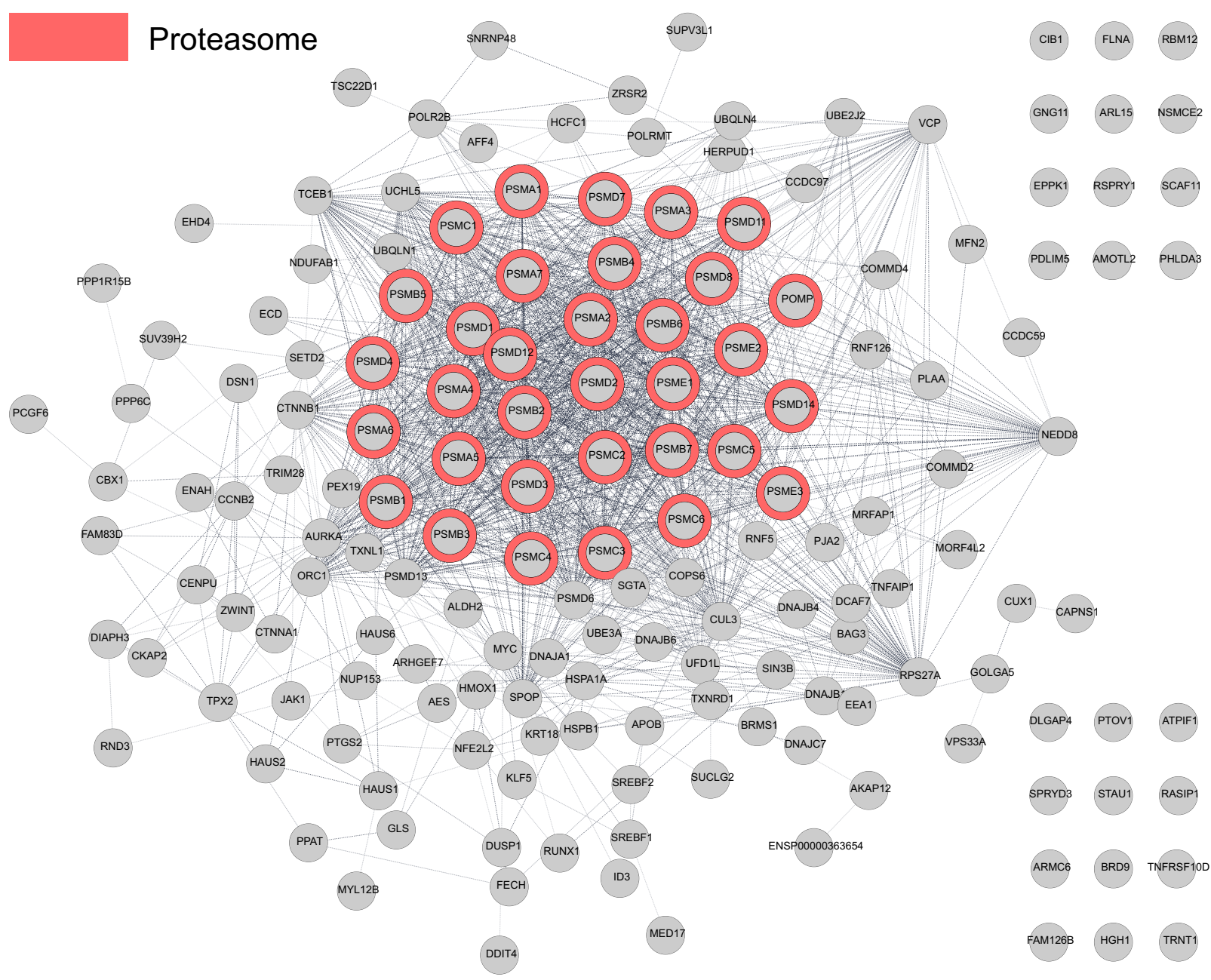

B

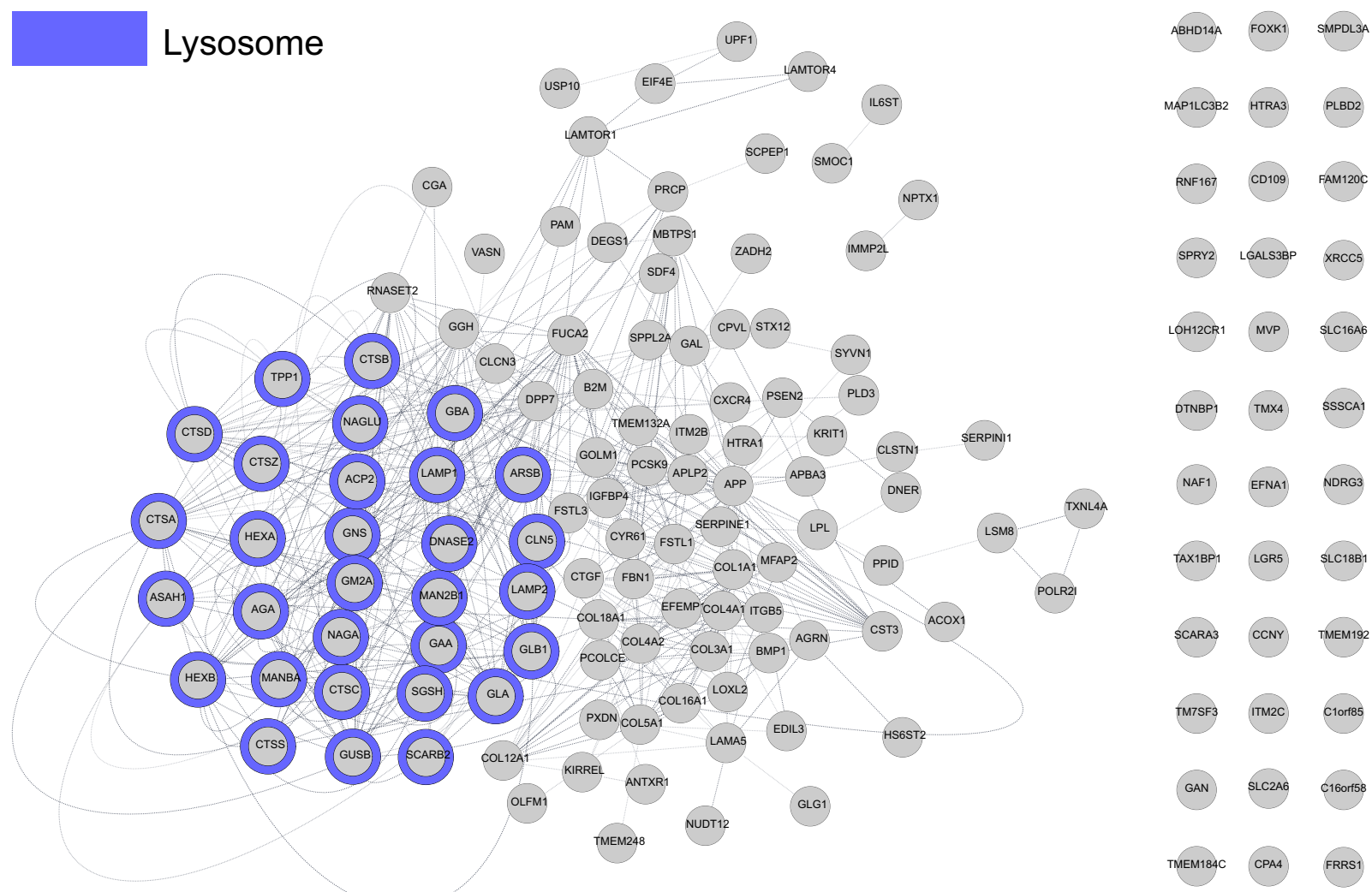

\section{Appendix Figure S6. Cargo analysis of autophagosomes upon proteasome inhibition.}

A. String analysis of proteins with enhanced abundance in autophagosomes upon MG132 treatment.

B. String analysis of proteins with reduced abundance in autophagosomes upon MG132 treatment. 
bioRxiv preprint doi: https://doi.org/10.1101/2021.04.16.440117; this version posted April 17, 2021. The copyright holder for this preprint (which was not certified by peer review) is the author/funder. All rights reserved. No reuse allowed without permission.

\begin{tabular}{|l|l|l|l|}
\hline ID & Pathway & Count & FDR \\
\hline hsa04142 & Lysosome & 30 & $3.02 \mathrm{e}-32$ \\
\hline hsa00511 & Other glycan degradation & 8 & $6.13 \mathrm{e}-10$ \\
\hline hsa00531 & Glycosaminoglycan degradation & 8 & $6.13 \mathrm{e}-10$ \\
\hline hsa04974 & Protein digestion and absorption & 8 & $1.95 \mathrm{e}-05$ \\
\hline hsa00603 & Glycoshingolipid biosynthesis & 4 & 0.00022 \\
\hline hsa00600 & Sphingolipid metabolism & 5 & 0.00062 \\
\hline hsa04512 & ECM-receptor interaction & 6 & 0.00073 \\
\hline hsa00604 & Glycosphingolipid biosynthesis - ganglio series & 3 & 0.0033 \\
\hline hsa05146 & Amoebiasis & 5 & 0.0104 \\
\hline hsa04933 & AGE-RAGE signaling pathway diabetic implications & 5 & 0.0111 \\
\hline hsa00052 & Galactose metabolism & 3 & 0.0194 \\
\hline hsa04210 & Apoptosis & 5 & 0.0354 \\
\hline hsa04145 & Phagosome & 5 & 0.0437 \\
\hline
\end{tabular}

\section{Appendix Figure S7. Pathways analysis of proteins with reduced appearance in autophagosomes upon MG132 treatment.}

KEGG pathway analysis of proteins with reduced autophagosome levels upon MG132 treatment. Pathways are presented with the number of proteins found in in the data set and computed FDRs for enrichment. 